Pacific Journal of Mathematics

SOME NEW RESULTS ON SIMPLE ALGEBRAS 


\section{SOME NEW RESULTS ON SIMPLE ALGEBRAS}

\section{J. RODABAUGH}

This paper deals with the problem of proving that a simple algebra (finite dimensional) has an identity element. The main result is contained in the following theorem. Let $A$ be a simple algebra (char. $\neq 2)$ in which $(x, x, x)=0$ and $x^{3} \cdot x=$ $x^{2} \cdot x^{2}$. If $M$ is a subset of $A$ such that $(A, M, A)=0$ and $(M, A, A) \cup(M, A) \cup(A, A, M) \subseteq M$, then $M=0$ or there is an identity element in $A$. This result is then used to prove the three following corollaries (char. $\neq 2)$ : (1) A simple power associative algebra with all commutators in the nucleus has an identity; (2) A simple power associative algebra with all associators in the middle center has an identity; (3) A simple antiflexible algebra in which $(x, x, x)=0$ and $A^{+}$is not nil has an identity.

For convenience in terminology, we define an algebra as a finite dimensional vector space on which a multiplication is defined that satisfies both distributive laws. An algebra is nilpotent if there is an integer $k$ such that any product of $k$ elements, no matter how associated, is zero. An element $x$ in an algebra is nilpotent if the subalgebra generated by $x$ is nilpotent. An algebra is nil if it consists entirely of nilpotent elements. A simple algebra is an algebra without proper ideals that is not nil. For char. $\neq 2$, define $x \cdot y=1 / 2(x y+y x)$. The algebra $A^{+}$is defined to be the vector space $A$ with miltiplication $x \cdot y$. In addition, define the commutator $(x, y)=x y-y x$ and the associator $(x, y, z)=(x y) z-x(y z)$. Using techniques similar to [3] we will prove the following theorem.

THEOREM 1. If $B$ is a subspace of an algebra $A$, there cannot be a nil subset $M$ proper in $B$ with:

(a) $B=M+M B$

(b) $M$ a subalgebra

(c) $(M, M, B)=0$.

Define $x^{1}=x$ and for $k>1$ define $x^{k}=x^{k-1} x$. Using this theorem, the following theorem is proved.

THEOREM 2. Let $A$ be a simple algebra (char. $\neq 2$ ) in which

Received June 29, 1964 and in revised form December 2, 1964. Presented in parts to the American Mathematical Society; October 21, 1963 under the title, "Algebras with all commutators in the center", and, December 26, 1963 under the title, "Some new results on simple algebras". 
$(x, x, x)=0$ and $x^{3} \cdot x=x^{2} \cdot x^{2}$. If $M$ is a subset of $A$ such that

(a) $(M, A, A) \subseteq M$

(b) $(M, A) \subseteq M$

(c) $(A, A, M) \subseteq M$

(d) $(A, M, A)=0$

then $M=0$ or there is an identity element in $A$.

It should be remarked that, if (d) could be replaced by $(A, M, A) \subseteq$ $M$, then $M=A$ would satisfy (a), (b), (c) and (d) in any algebra.

Many simple algebras are known to have identities. If the center (the set of $x$ such that $(x, y)=(x, y, z)=(y, z, x)=0$ for all $y$ and $z$ in $A$ ) of a simple algebra is not zero, the algebra has an identity element [4]. In addition, there are many identities which, if satisfied by a simple algebra, force that algebra to have an identity element.

In $\S 4$, Theorem 2 will be used to prove the existence of an identity element in three classes of algebras.

The author would like to thank the referee for suggesting conditions (a), (b), (c) and (d) in a more general form than was first submitted.

2. Proof of the first theorem. Let us assume that $M$ is a subset proper in $B$ that satisfies (a), (b) and (c) of Theorem 1 . We will show that $M$ is not nil. From (c), it is clear that $M$ is an associative subalgebra even though $A$ is not even assumed to be power associative. The following fact is easily obtained by induction. In an associative algebra such as $M, \Pi_{i=1}^{n} a_{i}=a_{1} \cdots a_{n}$.

LEMMA 2.1. If $a_{1}, \cdots, a_{n}$ are in $M$ and if $x$ is in $B$ then $\left(\prod_{i=1}^{n} a_{i}\right) x=a_{1}\left(a_{2} \cdots\left(a_{n} x\right) \cdots\right)$.

LEMmA 2.2. There exists an $x \neq 0$ in $B$ and an $a$ in $M$ with $x=a x$.

Proof. For any $x$ in $B \cap M^{\prime}$ (set theoretic complement), there is a set of elements $a_{1}, \cdots, a_{n}, b$ in $M$ and a set $y_{1}, \cdots, y_{n}$ in $B \cap M^{\prime}$ with $x=b+\sum_{i=1}^{n} a_{i} y_{i}$. Throughout this proof we will use $a, b$ with subscripts to indicate members of $M$ and $y$ with subscripts to indicate members of $B \cap M^{\prime}$. Since $M$ is proper in $B$, we have $B \cap M^{\prime} \neq \varnothing$. Let $y_{0}$ be in $B \cap M^{\prime}$.

Clearly, $y=\sum_{i=1}^{n_{1}} a_{1 i} y_{1 i}+b_{1}$. Since $y_{0}$ is not in $M$, some $j$ exists for which $a_{1 j} y_{1 j}$ is not in $M$. Define $y_{1}=y_{1 j}$ and $a_{1}=a_{1 j}$. Suppose 
$a_{1}, \cdots, a_{k}, y_{1}, \cdots, y_{k}$ have been defined for all $k<m$ with $a_{1}\left(a_{2} \cdots\left(a_{k} y_{k}\right) \cdots\right)$ in $B \cap M^{\prime}$. Since $y_{m-1}$ is not in $M, y_{m-1}=\sum_{i=1}^{n} a_{m i} y_{m i}+b_{m}$. There must be some $j$ for which $a_{1}\left(a_{2} \cdots\left(a_{m-1}\left(a_{m j} y_{m j}\right)\right) \cdots\right)$ is in $B \cap M^{\prime}$ because $a_{1}\left(a_{2} \cdots\left(a_{m-1} y_{m-1}\right) \cdots\right)$ is in $B \cap M^{\prime}$ and $M$ is a subalgebra. Defining $y_{m}=y_{m j}$ and $a_{m}=a_{m j}$, we have inductively defined for all $n$ the set $y_{1}, \cdots, y_{n}, a_{1}, \cdots, a_{n}$ with $\left(\prod_{i=1}^{n} a_{i}\right) y_{n}=a_{1}\left(a_{2} \cdots\left(a_{n} y_{n}\right) \cdots\right)$ in $B \cap M^{\prime}$. For $j<k$, let $m_{j k}=\prod_{i=j}^{k} a_{i}$. Also, let $r_{j}=\prod_{i=1}^{j} a_{i}$ so that when $1<j<k, r_{j-1} m_{j k}=r_{k}$. From finite dimensionality, there is an $s$ with $m_{1 s} y_{s}, \cdots, m_{s s} y_{s}$ a linearly dependent set. If $m_{j_{s}} y_{s}$ were in $M$ for $j>1$, then $r_{j-1}\left(m_{j s} y_{s}\right)=r_{s} y_{s}$ would be in $M$. This is not true by the construction of $y_{s}$ so $m_{j s} y_{s}$ is not in $M$ and hence is not zero for all $j$. Because the elements $m_{1 s} y_{s}, \cdots, m_{s s} y_{s}$ are dependent, there exist field elements $\alpha_{1}, \cdots, \alpha_{s}$ not all zero with $\sum_{i=1}^{s} \alpha_{i} m_{i s} y_{s}=0$. Letting: $t=\max \left\{i: a_{i} \neq 0\right\}$ will give

$$
m_{t s} y_{s}=-\sum_{i=1}^{t-1}\left(\alpha_{i} / \alpha_{t}\right) m_{i s} y_{s}=-\left[\sum_{i=1}^{t-1}\left(\alpha_{i} / \alpha_{t}\right) m_{i, t-1}\right] m_{t s} y_{s} .
$$

Letting $x=m_{t s} y_{s}$ and $a=-\sum_{i=1}^{t-1}\left(\alpha_{i} / \alpha_{t}\right) m_{i, t-1}$, will give $x=a x$ with $x \neq 0$ in $B$ and $a$ in $M$.

We are now able to prove that $M$ is not nil. By induction $x=$ $a x=a(a x)=a^{2} x=\cdots=a^{k} x$ for all $k$. Since $x$ is not zero, $a$ is not nilpotent and $M$ is not nil. Since $M$ is an associative algebra, we have the following result.

Corollary. If $B$ is a subspace of an algebra $A$ and if $M$ is a proper subset of $B$ with:

(a) $M+M B=B$

(b) $M$ a subalgebra

(c) $(M, M, B)=0$

then there is an idempotent in $M$.

3. Proof of the second theorem. Throughout this section we will assume that $A$ is a simple algebra in which $(x, x, x)=0$ and $x^{3} \cdot x=x^{2} \cdot x^{2}$. In addition, assume char. $\neq 2$. Given a subset of $A$ that satisfies (a), (b), (c) and (d) of Theorem, it is necessary to find a subalgebra of $A$ satisfying the same conditions.

Definition 3.1. The set $\mathscr{N}=$ subsets of $A$ that satisfy (a), (b), (c) and (d) of Theorem 2 .

Lemma 3.1. If $M$ is in $\mathscr{N}$ then either $M=0$ or $M+M A=A$.

This lemma is obvious for $M+M A$ is an ideal in $A$. From now 
on, assume that $\mathscr{N}$ contains a nonzero member. Each member of $\mathscr{N}$ can easily be extended to a subspace of $A, \mathscr{N}$ is closed under set union, and $A$ is finite dimensional. Consequently, there is a largest member of $\mathscr{N}$ which we will call $N$.

LEMma 3.2. The set $N$ is an associative subalgebra with $N+$ $N A=A$.

Proof. Clearly $N$ is a nonzero subspace so $N+N A=A$. In any ring, the following identities are satisfied:

$$
\begin{gathered}
(x y, z)+(y z, x)+(z x, y)=(x, y, z)+(y, z, x)+(z, x, y) \\
(w x, y, z)-(w, x y, z)+(w, x, y z)=w(x, y, z)+(w, x, y) z .
\end{gathered}
$$

If $x$ and $y$ are in $N$, then from (1), we have

$$
(x y, z)=(y, z, x)-(y z, x)-(z x, y) .
$$

Hence, $(x y, z)$ is in $N$. Equation (2) implies the fact that $(w, x y, z)=0$. If $w$ and $x$ are in $N$, then $(w x, y, z)=(w, x y, z)+w(x, y, z)$ which is in $N+N^{2}$. Also, $(x, y, x w)$ is in $N+N^{2}$. Consequently, $N+N^{2}$ is in $\mathscr{N}$ so $N+N^{2} \subseteq N$. The fact that $N$ is associative is obvious.

The proof of Lemma 3.2 can be used to construct a subalgebra $P$ satisfying (a), (b), (c) and (d) of Theorem 2 in a quite different way. First, extend $M$ to a subspace of $A$ and call this subspace $M$. Then for $k \geqq 1$, define $M_{k}=M_{k-1}^{2}+M_{k-1}$. Induction and the proof of Lemma 3.2 imply $M_{k}$ satisfies (a), (b), (c) and (d) of Theorem 2 for all $k$. By finite dimensionality, for some $k, M_{k}=M_{k+1}$. The set $P=M_{k}$ is a subalgebra satisfying (a), (b), (c) and (d). It is clear that $P+P A=A$.

We know that an associative simple algebra has an identity element. Therefore, if $A=N$, then $A$ has an identity element. From now on, assume $A \neq N$. Theorem 1 implies $N$ is not nil so there is an idempotent in $N$. An idempotent $e$ is principal if there is no idempotent $g$ with $e g=g e=0$. Because $N$ is associative, there is an idempotent that is principal in $N$. We will call this idempotent $f$. It cannot be assumed that $f$ is principal in $A$. We will need the following definition.

Definition 3.2. For any subset $B$ of $A, B_{i j}(e)=\{x$ in $B$ such that $e x=i x$ and $x e=j x\}$. Define $B_{i j}$ by $B_{i j}=B_{i j}(f)$.

Because $N$ is an associative algebra with $f$ in $N, N=N_{11}+N_{10}+$ $N_{01}+N_{00}$. In addition, $N_{i j} N_{j k} \subseteq N_{i k}$ and, for $j \neq k, N_{i j} N_{k m}=0$. Up to now, we have not used the fact that $(x, x, x)=0$. Linearizing this 
yields this multilinear identity:

$$
\begin{gathered}
(x, y, z)+(y, z, x)+(z, x, y)+(z, y, x) \\
+(y, x, z)+(x, z, y)=0 .
\end{gathered}
$$

If we now let $x=z=f$ and divide by 2 , we obtain $(f, y, f)=0$. Therefore, we have $(f, f, y)=(f, y, f)=(y, f, f)=0$ which is all that is needed to prove this lemma:

Lemma 3.3. Relative to $f, A=A_{11}+A_{10}+A_{01}+A_{00}$.

Lemma 3.4. The subspaces $N_{i j}$ and $A_{i j}$ obey the following laws:

$$
\begin{gathered}
A_{i j} A_{k m}=0 \text { if } j \neq k \\
N_{i j} A_{j k} \cup A_{i j} N_{j k} \subseteq N_{i k} \text { if } i \neq k \\
N_{i j} A_{j k} \subseteq A_{i 1}+A_{i 0} \\
A_{i j} N_{j k} \subseteq A_{1 k}+A_{0 k} \\
N_{10} A_{01} \subseteq N_{11}+N_{10} \\
N_{01} A_{10} \leqq N_{00}+N_{01} \\
N_{i i} A_{i i} \subseteq A_{i i}+N .
\end{gathered}
$$

Proof. To prove (5), let $x$ be in $A_{i j}$ and let $y$ be in $A_{k m}$ in $(x, f, y)=0$. The relations (7) and (8) result from $\left(f, N_{i j}, A_{j_{k}}\right)=0$ and $\left(A_{i j}, N_{j k}, f\right)=0$. In proving (6), we first let $y=f, z=n_{i j}$ (in $N_{i j}$ ) and $x=x_{j k}$ (in $A_{j k}$ ) in (4) to obtain $\left(f, x_{j k}, n_{i j}\right)+\left(n_{i j}, x_{j k}, f\right)=0$. Using (5), we find that $\left(f, x_{j k}, n_{i j}\right)=j x_{j k} n_{i j}-f\left(x_{j k} n_{i j}\right)=0$. Hence, $\left(n_{i j} x_{j k}\right) f=k n_{i j} x_{j k}$. This together with (7) implies $N_{i j} A_{j k} \subseteq A_{i k}$. In a similar way, (4), (5) and (8) imply $A_{i j} N_{j k} \subseteq A_{i k}$. We will now prove $N_{i j} A_{j k} \subseteq N_{i k}$. The proof of $A_{i j} N_{j k} \subseteq N_{j k}$ is similar. Using $z$ and $x$ as defined above, $x z=0$ and $(z, x)$ is in $N$. Therefore $z x=(z, x)$ is in $N \cap A_{i k}$ and we have proved (6). We now take $n$ in $N_{10}$ and $a$ in $A_{01}$. From (7) and (8) we have $n a=b_{11}+b_{10}$ and $a n=c_{10}+c_{00}$ with $b_{i j}, c_{i j}$, in $A_{i j}$. We also see that $b_{11}, c_{11}$ and $b_{10}-c_{10}$ are in $N$ because $(n, a)$ is in $N$. Equation (4) implies $(n, a, f)+(f, a, n)=0$. Upon substitution we get $\left(b_{11}+b_{10}\right) f-\left(b_{11}+b_{10}\right)-f\left(c_{10}+c_{00}\right)=0$ or $-b_{10}-c_{10}=$ 0. Therefore $b_{10}=1 / 2\left(b_{10}-c_{10}\right)$ is in $N_{10}$. This proves (9) and a similar argument proves (10). In [1], a linearization of $\left(x^{2} \cdot x\right) \cdot x=x^{2} \cdot x^{2}$ was all that was needed to prove that $A_{11} \cdot A_{11} \subseteq A_{11}$ and $A_{00} \cdot A_{00} \subseteq A_{00}$. The proof of Lemma 3.4 is completed by observing that this implies $N_{i i} A_{i i} \subseteq$ $N_{i i} \cdot A_{i i}+\left(N_{i i}, A_{i i}\right) \subseteq A_{i i}+N$.

Lemma 3,5, The algebra $A=N+N_{11} A_{11}+N_{00} A_{00}$. 
Proof. As a result of (5) and Lemma 3.2, $A=N+\sum N_{i j} A_{j k}$. From (6), $N_{11} A_{10}+N_{10} A_{00} \subseteq N_{10}$ and $N_{00} A_{01}+N_{01} A_{11} \subseteq N_{01}$. The relations in (9) and (10) complete the proof.

Lemma 3.6. The set $B=N_{10} N_{01}+N_{10}+N_{01}+N_{01} N_{10}$ is an associative ideal in $A$.

Proof. The laws $N_{i j} N_{j k} \subseteq N_{i k}$ and $N_{i j} N_{m k}=0$ for $j \neq m$ easily establish the fact that $B$ is an associative ideal in $N$. Using Lemma 3.5 , we need only prove that $B\left(N_{11} A_{11}\right),\left(N_{11} A_{11}\right) B,\left(N_{00} A_{00}\right) B$ and $B\left(N_{00} A_{00}\right)$ are in $B$. We have $\left(N_{11} A_{11}\right)\left(N_{10}+N_{01}\right) \subseteq\left(A_{11}+N\right)\left(N_{10}+N_{01}\right) \subseteq A_{11} N_{10}+$ $B \subseteq B$. Also, $\left(N_{10}+N_{01}\right)\left(N_{11} A_{11}\right)=\left[\left(N_{10}+N_{01}\right) N_{11}\right] A_{11} \subseteq N_{01} A_{11} \subseteq N_{01} \subseteq$ $B$. Interchanging 0 and 1 will prove that $\left(N_{00} A_{00}\right)\left(N_{10}+N_{01}\right)$ and $\left(N_{01}+N_{01}\right)\left(N_{00} A_{00}\right)$ are in $B$. Therefore $\left(N_{10}+N_{01}\right) A$ and $A\left(N_{10}+N_{01}\right)$ are in $B$. Now, $\left(N_{10} N_{01}\right) A=N_{10}\left(N_{01} A\right) \leqq N_{10} B \cong B$ and $A\left(N_{10} N_{01}\right)=$ $\left(A N_{10}\right) N_{01} \subseteq B N_{01} \subseteq B$. Similarly $\left(N_{01} N_{10}\right) A$ and $A\left(N_{01} N_{10}\right)$ are in $B$ so $B$ is an associative ideal in $A$.

We have assumed that $N \neq A$ which implies $B=0$. Therefore $A=N_{11}+N_{11} A_{11}+N_{00}+N_{00} A_{00}$. The relations in (7) and (11) together with the fact that $N=N_{11}+N_{00}$ imply $N_{11} A_{11} \subseteq A_{11}$ and $N_{00} A_{00} \subseteq A_{00}$.

We are now able to complete the proof of Theorem 2. We have $A=A_{11}+A_{00}$ with $A_{11}=N_{11}+N_{11} A_{11}$ and $A_{00}=N_{00}+N_{00} A_{00}$. The result in Theorem 1 and the fact that $N_{00}$ is nil imply $N_{00}=A_{00}$. Therefore, using (5) and $N_{00}^{2} \subseteq N_{00}$, we see that $N_{00}$ is an ideal of $A$. Consequently, $f$ is the identity element of $A$.

4. Applications, We will mention several cases where Theorem 2 can be used to prove the existence of an identity element in a simple algebra. The following two corollaries are obvious since simple associative and simple commutative power associative algebras have identities. The nucleus of an algebra $A$ is $\{x: x y=y x$ for all $y$ in $A\}$.

CoRollary 4.1. For char. $\neq 2$, a simple power associative algebra with all commutators in the nucleus has an identity element.

COROLlARY 4.2. For char. $\neq 2$ a simple power associative algebra with all associators in the middle center $(\{x:(x, y)=(y, x, z)=0$ for all $y$ and $z\}$ ) has an identity element.

For our last application, let $A$ be a simple algebra that satisfies the following:

$$
(x, y, z)=(z, y, x) \quad(\text { the antiflexible law })
$$




$$
(x, x, x)=0 \text {. }
$$

In [2] it is proved that $x^{2} x^{2}=x^{3} x=x x^{3}$ in $A$. In fact, $A^{+}$is power associative [2]. Furthermore, if we let $Z=\{x: x y=y x$ for all $y$ in $A\}$, we can show by the arguments of [3] that $(A, Z, A)=0$ and $(Z, A, A)$, $(A, A, Z)$ are subsets of $Z$. Because $A^{+}$is power associative, there is an idempotent $e$ in $A^{+}$if $A^{+}$is not nil. Clearly, $e$ is an idempotent in $A$. The arguments of [2] will show $A=A_{11}(e)+A_{00}(e)$. Therefore, $e$ is in $Z$ and $Z \neq 0$. We have proved this corollary.

Corollary 4.3. If $A$ is a simple antiflexible algebra (char. $\neq 2$ ) that satisfies $(x, x, x)=0$ and if $A^{+}$is not a nil algebra, then there is an identity element in $A$.

\section{BIBLIOGRAPHY}

1. A. A. Albert, Power associative rings, Trans. Amer. Math. Soc. 64, No. 3 (1948), $552-593$.

2. F. Kosier, On a class of nonflexible algebras, Trans. Amer. Math. Soc. 102, No. 2 (1962), 299-318.

3. D. Rodabaugh, A generalization of the flexible law, accepted for publication by Trans. Amer. Math. Soc.

4. R. D. Schafer, An Introduction to Nonassociative Algebras, Oklahoma State University (1961).

VANDERBILT UNIVERSITY 



\section{PACIFIC JOURNAL OF MATHEMATICS}

\section{EDITORS}

\author{
H. SAMELSON \\ Stanford University \\ Stanford, California \\ R. M. BLUMENTHAL \\ University of Washington \\ Seattle, Washington 98105
}

\author{
*J. DUGUNDJI \\ University of Southern California \\ Los Angeles, California 90007
}

RICHARD ARENS

University of California Los Angeles, California 90024

\section{ASSOCIATE EDITORS}
E. F. BECKENBACH
B. H. NEUMANN
F. WOLF
K. YoSIDA

\section{SUPPORTING INSTITUTIONS}

\author{
UNIVERSITY OF BRITISH COLUMBIA \\ CALIFORNIA INSTITUTE OF TECHNOLOGY \\ UNIVERSITY OF CALIFORNIA \\ MONTANA STATE UNIVERSITY \\ UNIVERSITY OF NEVADA \\ NEW MEXICO STATE UNIVERSITY \\ OREGON STATE UNIVERSITY \\ UNIVERSITY OF OREGON \\ OSAKA UNIVERSITY \\ UNIVERSITY OF SOUTHERN CALIFORNIA
}

\author{
STANFORD UNIVERSITY \\ UNIVERSITY OF TOKYO \\ UNIVERSITY OF UTAH \\ WASHINGTON STATE UNIVERSITY \\ UNIVERSITY OF WASHINGTON \\ * * * * \\ AMERICAN MATHEMATICAL SOCIETY \\ CHEVRON RESEARCH CORPORATION \\ TRW SYSTEMS \\ NAVAL ORDNANCE TEST STATION
}

Mathematical papers intended for publication in the Pacific Journal of Mathematics should be typewritten (double spaced). The first paragraph or two must be capable of being used separately as a synopsis of the entire paper. It should not contain references to the bibliography. Manuscripts may be sent to any one of the four editors. All other communications to the editors should be addressed to the managing editor, Richard Arens at the University of California, Los Angeles, California 90024.

50 reprints per author of each article are furnished free of charge; additional copies may be obtained at cost in multiples of 50 .

The Pacific Journal of Mathematics is published monthly. Effective with Volume 16 the price per volume (3 numbers) is $\$ 8.00$; single issues, $\$ 3.00$. Special price for current issues to individual faculty members of supporting institutions and to individual members of the American Mathematical Society: $\$ 4.00$ per volume; single issues $\$ 1.50$. Back numbers are available.

Subscriptions, orders for back numbers, and changes of address should be sent to Pacific Journal of Mathematics, 103 Highland Boulevard, Berkeley 8, California.

Printed at Kokusai Bunken Insatsusha (International Academic Printing Co., Ltd.), No. 6, 2-chome, Fujimi-cho, Chiyoda-ku, Tokyo, Japan.

\section{PUBLISHED BY PACIFIC JOURNAL OF MATHEMATICS, A NON-PROFIT CORPORATION}

The Supporting Institutions listed above contribute to the cost of publication of this Journal, but they are not owners or publishers and have no responsibility for its content or policies.

* Paul A. White, Acting Editor until J. Dugundji returns. 


\section{Pacific Journal of Mathematics}

\section{Vol. 17, No. 2 \\ February, 1966}

Henry A. Antosiewicz, Boundary value problems for nonlinear ordinary

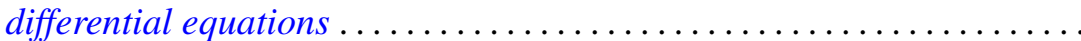

Bernard Werner Levinger and Richard Steven Varga, Minimal Gerschgorin sets. II ........................................

Paul Camion and Alan Jerome Hoffman, On the nonsingularity of complex matrices........................................ 211

J. Chidambaraswamy, Divisibility properties of certain factorials ........ 215

J. Chidambaraswamy, A problem complementary to a problem of Erdös .... 227

John Dauns, Chains of modules with completely reducible quotients ...... 235

Wallace E. Johnson, Existence of half-trajectories in prescribed regions and asymptotic orbital stability ............................ 243

Victor Klee, Paths on polyhedra. II . ........................ 249

Edwin Haena Mookini, Sufficient conditions for an optimal control problem in the calculus of variations ............................ 263

Zane Clinton Motteler, Existence theorems for certain quasi-linear elliptic equations........................................... 279

David Lewis Outcalt, Simple n-associative rings ............... 301

David Joseph Rodabaugh, Some new results on simple algebras ......... 311

Oscar S. Rothaus, Asymptotic properties of groups generation ........... 319

Ernest Edward Shult, Nilpotence of the commutator subgroup in groups admitting fixed point free operator groups .............

William Hall Sills, On absolutely continuous functions and the

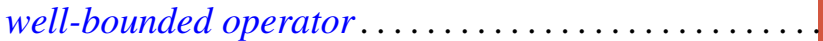

Joseph Gail Stampfli, Which weighted shifts are subnormal ..

Donald Reginald Traylor, Metrizability and completeness in normal Moore spaces 